\title{
EXISTENCE OF HOMOCLINIC SOLUTIONS FOR SECOND ORDER HAMILTONIAN SYSTEMS UNDER LOCAL CONDITIONS
}

\author{
LI-LI WAN
}

Abstract. Under some local conditions on $V(t, x)$ with respect to $x$, the existence of homoclinic solutions is obtained for a class of the second order Hamiltonian systems $\ddot{u}(t)+\nabla V(t, u(t))=$ $f(t), \forall t \in \mathbb{R}$.

Mathematics subject classification (2010): 34C37, 37J45.

Keywords and phrases: homoclinic solutions, second order Hamiltonian systems, local conditions.

\section{REFERENCES}

[1] V. Coti-Zelati And P. H. Rabinowitz, Homoclinic orbits for second order Hamiltonian systems possessing superquadratic potentials, J. Amer. Math. Soc., 4, (1991), 693-727.

[2] Y. H. DING, Existence and multiplicity results for homoclinic solutions to a class of Hamiltonian systems, Nonlinear Anal., 25, (1995), 1095-1113.

[3] A. DAOUAS, Homoclinic orbits for superquadratic Hamiltonian systems without a periodicity assumption, Nonlinear Anal., 74, (2011), 3407-3418.

[4] M. IZYDOREK AND J. JANCZEWSKA, Homoclinic solutions for a class of the second order Hamiltonian systems, J. Differential Equations, 219, (2005), 375-389.

[5] P. Korman AND A. C. LAZER, Homoclinic solutions for a class of second order Hamiltonian systems, Electron. J. Differential Equations, 1, (1994), 1-10.

[6] X. LV AND J. JiAng, Existence of homoclinic solutions for a class of second-order Hamiltonian systems with general potentials, Nonlinear Anal., 13, (2012), 1152-1158.

[7] S. LU, Homoclinic solutions for a nonlinear second order differential system with p-Laplacian operator, Nonlinear Anal., 12, (2011), 525-534.

[8] J. Mawhin And M. Willem, Critical Point Theory and Hamiltonian Systems, Springer, New York, 1989.

[9] P.H. RABINOWITZ AND K. TANAKA, Some results on connecting orbits for a class of Hamiltonian systems, Math. Z., 206, (1990), 473-499.

[10] J. SUn AND T. WU, Multiplicity and concentration of homoclinic solutions for some second order Hamiltonian system, Nonlinear Anal., 114, (2015), 105-115.

[11] X.H. TANG AND Li XIAO, Homoclinic solutions for a class of second-order Hamiltonian systems, Nonlinear Anal., 71, (2009), 1140-1152.

[12] X.H. TANG AND X. Lin, Homoclinic solutions for a class of second-order Hamiltonian systems, J. Math. Anal. Appl., 354, (2009), 539-549.

[13] D.L. WU, X.P. WU AND C.L. TANG, Homoclinic solutions for a class of nonperiodic and noneven second-order Hamiltonian systems, J. Math. Anal. Appl., 367, (2010), 154-166.

[14] J. YANG AND F.B. ZHANG, Infinitely many homoclinic orbits for the second-order Hamiltonian systems with super-quadratic potentials, Nonlinear Anal., 10, (2009), 1417-1423.

[15] M.H. YANG AND Z.Q. HAN, Infinitely many homoclinic solutions for second-order Hamiltonian systems with odd nonlinearities, Nonlinear Anal., 74, (2011), 2635-2646.

[16] Y. YE AND C. L. TANG, Multiple homoclinic solutions for second-order perturbed Hamiltonian systems, Studies in Applied Math., 132, (2014), 112-137.

[17] W. M. ZOU AND S. J. LI, Infinitely many homoclinic orbits for the second-order hamiltonian systems, Appl. Math. Lett., 16, (2003), 1283-1287. 
[18] Z. ZHANG AND R. YUAN, Homoclinic solutions for some seconde order non-autonomous Hamiltonian systems without the globally superquadratic condition, Nonlinear Anal., 72, (2010), 1809-1819.

[19] Z. ZHANG, T. XIANG AND R. YUAN, Homoclinic solutions for subquadratic Hamiltonian systems without coercive conditions, Taiwanese Journal of Math., 18, (2014), 1089-1105.

[20] Q.Y. ZHANG AND L.P. CHU, Homoclinic solutions for a class of second order Hamiltonian systems with locally defined potentials, Nonlinear Anal., 75, (2012), 3188-3197. 